\title{
Hidden beauty molecules with the local hidden gauge approach and heavy quark spin symmetry
}

\author{
Chu-Wen Xiao*i \\ Departamento de Física Teórica and IFIC, Centro Mixto Universidad de Valencia-CSIC, \\ Institutos de Investigación de Paterna, Apartado 22085, 46071 Valencia, Spain \\ E-mail: खiaochwdific.uv.es
}

Altug Ozpineci

Physics Department, Middle East Technical University, 06531 Ankara, Turkey

E-mail: ozpineciemetu.edu.tr

\section{Eulogio Oset}

Departamento de Física Teórica and IFIC, Centro Mixto Universidad de Valencia-CSIC, Institutos de Investigación de Paterna, Apartado 22085, 46071 Valencia, Spain

E-mail: esetaific.uv.es

Using a coupled channel unitary approach, combining the heavy quark spin symmetry and the dynamics of the local hidden gauge, we investigate the meson-meson interaction with hidden beauty. We have investigated both $I=0$ and $I=1$ states, and obtain several new states of isospin $I=0$ : six bound states, and weakly bound six more possible states which depend on the influence of the coupled channel effects. But there is no state found in the $I=1$ sector since the interactions are too weak to create any bound states within our framework.

XV International Conference on Hadron Spectroscopy-Hadron 2013

4-8 November 2013

Nara, Japan

\footnotetext{
*Presenter

${ }^{\dagger}$ Thanks for the local support of the organizer.
} 


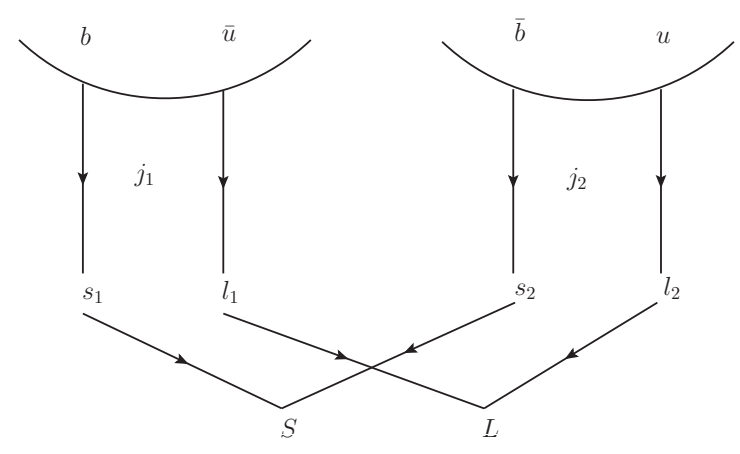

Figure 1: Diagrams for the hidden beauty systems.

\section{Introduction}

The world of heavy quarks, charm and beauty, is experiencing a fast development, with a plethora of new states being found in facilities as BABAR, CLEO, BELLE, BES. Recently, the discovery of the hidden beauty $Z_{b}(10610)$ and $Z_{b}(10650)$ states [四, has driven more attention to the beauty sector [1], 目].

In this work, we investigate the hidden beauty system of meson-meson interaction [ [ 9 , [1, 目].

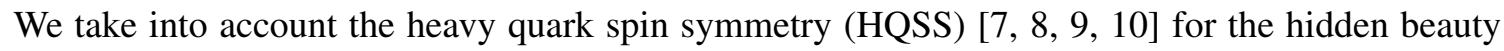
sector, and then, under the lower order HQSS constrain, we use the local hidden gauge approach [1, [2] to determine the interaction potentials.

\section{Formalism}

In our work, we use the coupled channel approach to study the meson-meson interaction in the hidden beauty sector, with the coupled channels of $B_{(s)}^{(*)} \bar{B}_{(s)}^{(*)}:(1) J=0, I=0, B \bar{B}, B_{s} \bar{B}_{s}, B^{*} \bar{B}^{*}$, $B_{s}^{*} \bar{B}_{s}^{*}$; (2) $J=0, I=1 B \bar{B}, B^{*} \bar{B}^{*}$; (3) $J=1, I=0, B \bar{B}^{*}\left(B^{*} \bar{B}\right), B_{s} \bar{B}_{s}^{*}\left(B_{s}^{*} \bar{B}_{s}\right), B^{*} \bar{B}^{*}, B_{s}^{*} \bar{B}_{s}^{*}$; (4) $J=1, I=1, B \bar{B}^{*}\left(B^{*} \bar{B}\right), B^{*} \bar{B}^{*}$; (5) $J=2, I=0, B^{*} \bar{B}^{*}, B_{s}^{*} \bar{B}_{s}^{*}$; (6) $J=2, I=1, B^{*} \bar{B}^{*}$.

In our case, all the hidden beauty systems are made by a meson $(M)$ - antimeson $(\bar{M})$ state,

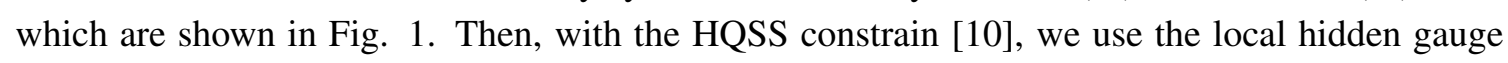
formalism to evaluate the interaction potential (more details, seen in our recent paper [[13]), following development of Refs. [144, [5]]. In principle one is using SU(4) symmetry to evaluate the couplings. However, recently we have shown in [16, [1]] that the leading terms respecting HQSS correspond in our approach to having the beauty quarks as spectators. In this case all couplings can be obtained using SU(3).

\section{Results}

We use the Bethe-Salpeter equation in coupled channels to evaluate the scattering amplitudes,

$$
T=[1-V G]^{-1} V .
$$


For the $G$ function, we take

$$
G(s)=\int \frac{d^{3} \vec{q}}{(2 \pi)^{3}} f^{2}(\vec{q}) \frac{\omega_{1}+\omega_{2}}{2 \omega_{1} \omega_{2}} \frac{1}{P^{02}-\left(\omega_{1}+\omega_{2}\right)^{2}+i \varepsilon} ; \quad f(\vec{q})=\frac{m_{V}^{2}}{\vec{q}^{2}+m_{V}^{2}},
$$

where $f(\vec{q})$ is the form factor, which comes from the light vector meson exchange.

Our results of the poles and the couplings for the $J^{P C}=2^{++}$channel with $q_{\max }=415 \mathrm{MeV}$ (left panel) and $q_{\max }=830 \mathrm{MeV}$ (right panel), are shown as Table $\mathbb{W}$. When ignoring the coupled channel effect, the results are shown in Table [D.

Table 1: The poles and couplings for the $J^{P C}=2^{++}: q_{\max }=415 \mathrm{MeV}$ (left panel) and $q_{\max }=830 \mathrm{MeV}$ (right panel), all units in $\mathrm{MeV}$.

\begin{tabular}{ccc|ccc}
\hline 10613 & $B^{*} \bar{B}^{*}$ & $B_{s}^{*} \bar{B}_{s}^{*}$ & 10469 & $B^{*} \bar{B}^{*}$ & $B_{s}^{*} \bar{B}_{s}^{*}$ \\
\hline$g_{i}$ & 86168 & 45864 & $g_{i}$ & 174393 & 92843 \\
\hline
\end{tabular}

Table 2: The poles and couplings for the $J^{P C}=2^{++}$ignoring coupled channels (two panels and units the same as before, also the same for below.

\begin{tabular}{ccc|ccc}
\hline 10616 & $B^{*} \bar{B}^{*}$ & $B_{s}^{*} \bar{B}_{s}^{*}$ & 10500 & $B^{*} \bar{B}^{*}$ & $B_{s}^{*} \bar{B}_{s}^{*}$ \\
\hline$g_{i}$ & 81595 & 0 & $g_{i}$ & 159102 & 0 \\
\hline 10828 & $B^{*} \bar{B}^{*}$ & $B_{s}^{*} \bar{B}_{s}^{*}$ & 10812 & $B^{*} \bar{B}^{*}$ & $B_{s}^{*} \bar{B}_{s}^{*}$ \\
\hline$g_{i}$ & 0 & 19787 & $g_{i}$ & 0 & 44102 \\
\hline
\end{tabular}

For the $J=1, I=0$ sector, the results with coupled channels and without coupled channels are shown in Tables B and 团.

Table 3: The poles and couplings for the $J^{P C}=1^{+-}$and $J^{P C}=1^{++}$.

\begin{tabular}{ccc|ccc}
\hline 10568 & $B \bar{B}^{*} \pm$ c.c. & $B_{s} \bar{B}_{s}^{*} \pm$ c.c. & 10425 & $B \bar{B}^{*} \pm$ c.c. & $B_{s} \bar{B}_{s}^{*} \pm$ c.c. \\
\hline$g_{i}$ & 85433 & 45560 & $g_{i}$ & 172908 & 92232 \\
\hline
\end{tabular}

Table 4: The poles and couplings for the $J^{P C}=1^{+-}$and $J^{P C}=1^{++}$ignoring coupled channels.

\begin{tabular}{ccc|ccc}
\hline 10571 & $B \bar{B}^{*} \pm$ c.c. & $B_{s} \bar{B}_{s}^{*} \pm$ c.c. & 10455 & $B \bar{B}^{*} \pm$ c.c. & $B_{s} \bar{B}_{s}^{*} \pm$ c.c. \\
\hline$g_{i}$ & 80884 & 0 & $g_{i}$ & 157691 & 0 \\
\hline 10783 & $B \bar{B}^{*} \pm$ c.c. & $B_{s} \bar{B}_{s}^{*} \pm$ c.c. & 10768 & $B \bar{B}^{*} \pm$ c.c. & $B_{s} \bar{B}_{s}^{*} \pm$ c.c. \\
\hline$g_{i}$ & 0 & 19611 & $g_{i}$ & 0 & 43776 \\
\hline
\end{tabular}

Finally, we get results for the $J^{P C}=0^{++}$sector as listing in Tables $\square$ and 6 . 
Table 5: The poles and couplings for the $J^{P C}=0^{++}$.

\begin{tabular}{ccc|ccc}
\hline 10523 & $B \bar{B}$. & $B_{s} \bar{B}_{s}$ & 10380 & $B \bar{B}$ & $B_{s} \bar{B}_{s}$ \\
\hline$g_{i}$ & 85045 & 45257 & $g_{i}$ & 172046 & 91591 \\
\hline
\end{tabular}

Table 6: The poles and couplings for the $J^{P C}=0^{++}$ignoring coupled channels.

\begin{tabular}{ccc|ccc}
\hline 10526 & $B \bar{B}$. & $B_{s} \bar{B}_{s}$ & 10410 & $B \bar{B}$ & $B_{s} \bar{B}_{s}$ \\
\hline$g_{i}$ & 80528 & 0 & $g_{i}$ & 156968 & 0 \\
\hline 10738 & $B \bar{B}$ & $B_{s} \bar{B}_{s}$ & 10723 & $B \bar{B}$ & $B_{s} \bar{B}_{s}$ \\
\hline$g_{i}$ & 0 & 19441 & $g_{i}$ & 0 & 43443 \\
\hline
\end{tabular}
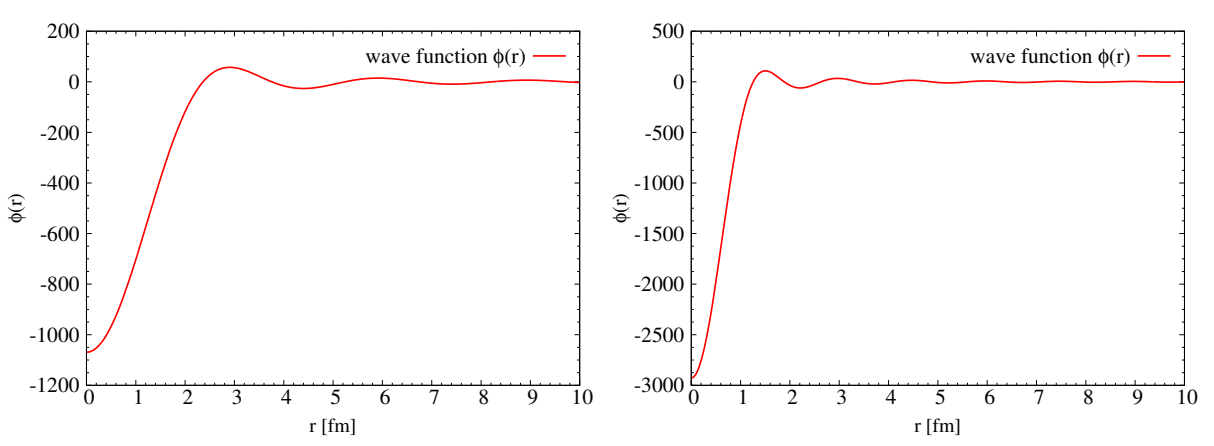

Figure 2: The wave functions of $B \bar{B}$ state, Left: $q_{\max }=415 \mathrm{MeV}$; Right: $q_{\max }=830 \mathrm{MeV}$.

\section{Discussions}

For a resonance or bound state, the sum rule [एष] is fulfilled: $P_{p}=-\sum_{i} g_{i}^{2}\left[\frac{d G_{i}}{d E}\right]_{E=E_{p}}=1$. For $B \bar{B}$ state, taking $q_{\max }=415 \mathrm{MeV}$, we get $P_{B \bar{B}}=0.985$, which means that the bound state is mostly made by $B \bar{B}$ with a minor $B_{s} \bar{B}_{s}$ component. This $B \bar{B}$ state is stable and independent of the free parameters of our formalism, which can be seen in Table $\square$.

Table 7: The poles in the $J^{P C}=0^{++}$channel when the cut off is changed (units in $\mathrm{MeV}$ ).

\begin{tabular}{|c|c|c|c|c|c|}
\hline$q_{\max }$ & 450 & 500 & 600 & 700 & 800 \\
\hline pole & 10513 & 10498 & 10464 & 10427 & 10389 \\
\hline
\end{tabular}

We also investigate the wave function and radius of the state. By performing some derivation, we get

$$
\phi(\vec{r})=\frac{1}{(2 \pi)^{3 / 2}} \frac{4 \pi}{r} \frac{1}{C} \int_{q_{\max }} p d p \sin (p r) \frac{\Theta\left(q_{\max }-|\vec{p}|\right)}{E-\omega_{1}(\vec{p})-\omega_{2}(\vec{p})} \frac{m_{V}^{2}}{\vec{q}^{2}+m_{V}^{2}},
$$

where we take $m_{V}=m_{\rho}=775 \mathrm{MeV}$. For the $B \bar{B}$ state, using Eq. (4⿴囗d), we show the results of wave function in Fig.D. The radii of the states are given in Table $\mathbb{8}$, which are of the same order of magnitude as Refs. [, [9]. 
Table 8: The radii of the states.

\begin{tabular}{|c|c|c|}
\hline states & $q_{\max }=415 \mathrm{MeV}$ & $q_{\max }=830 \mathrm{MeV}$ \\
\hline$B^{*} \bar{B}^{*}$ & $1.46 \mathrm{fm}$ & $0.72 \mathrm{fm}$ \\
\hline$B \bar{B}^{*}$ & $1.46 \mathrm{fm}$ & $0.72 \mathrm{fm}$ \\
\hline$B \bar{B}$ & $1.46 \mathrm{fm}$ & $0.72 \mathrm{fm}$ \\
\hline
\end{tabular}

\section{Conclusions}

In our work, combining the local hidden gauge symmetry with heavy quark spin symmetry, we investigate the hidden beauty sector: $B_{(s)}^{(*)} \bar{B}_{(s)}^{(*)}$. In the $I=0$ sector, we obtain 6 hidden beauty resonances with binding energies $34 \mathrm{MeV}(178 \mathrm{MeV})$ for $q_{\max }=415 \mathrm{MeV}(830 \mathrm{MeV})$, and 6 hidden beauty-hidden strange states with binding energies $2 \mathrm{MeV}(18 \mathrm{MeV})$. But, for the $I=1$ sector, the interaction is too weak to form any bound states. We hope that these states can be found in experiments in the future.

\section{References}

[1] A. Bondar et al. [Belle Collaboration], Phys. Rev. Lett. 108, 122001 (2012).

[2] Z. -F. Sun, J. He, X. Liu, Z. -G. Luo and S. -L. Zhu, Phys. Rev. D 84, 054002 (2011).

[3] M. Cleven, Q. Wang, F. -K. Guo, C. Hanhart, U. -G. Meissner and Q. Zhao, Phys. Rev. D 87, 074006 (2013).

[4] Y. -J. Zhang, H. -C. Chiang, P. -N. Shen and B. -S. Zou, Phys. Rev. D 74, 014013 (2006).

[5] S. Ohkoda, Y. Yamaguchi, S. Yasui, K. Sudoh and A. Hosaka, Phys. Rev. D 86, 034019 (2012).

[6] M. T. Li, W. L. Wang, Y. B. Dong and Z. Y. Zhang, J. Phys. G 40, 015003 (2013).

[7] N. Isgur and M. B. Wise, Phys. Lett. B 232, 113 (1989).

[8] M. Neubert, Phys. Rept. 245, 259 (1994).

[9] F. -K. Guo, C. Hanhart and U. -G. Meissner, Phys. Rev. Lett. 102, 242004 (2009).

[10] C. Garcia-Recio, V. K. Magas, T. Mizutani, J. Nieves, A. Ramos, L. L. Salcedo and L. Tolos, Phys. Rev. D 79, 054004 (2009).

[11] M. Bando, T. Kugo, S. Uehara, K. Yamawaki and T. Yanagida, Phys. Rev. Lett. 54, 1215 (1985).

[12] M. Bando, T. Kugo and K. Yamawaki, Phys. Rept. 164, 217 (1988).

[13] A. Ozpineci, C. W. Xiao and E. Oset, Phys. Rev. D 88, 034018 (2013).

[14] J. -J. Wu, R. Molina, E. Oset and B. S. Zou, Phys. Rev. Lett. 105, 232001 (2010).

[15] J. -J. Wu, R. Molina, E. Oset and B. S. Zou, Phys. Rev. C 84, 015202 (2011).

[16] W. H. Liang, C. W. Xiao and E. Oset, arXiv:1401.1441 [hep-ph], accepted by Phys. Rev. D.

[17] W. H. Liang, T. Uchino, C. W. Xiao and E. Oset, arXiv:1402.5293 [hep-ph].

[18] D. Gamermann, J. Nieves, E. Oset and E. Ruiz Arriola, Phys. Rev. D 81, 014029 (2010).

[19] M. T. Li, W. L. Wang, Y. B. Dong and Z. Y. Zhang, Int. J. Mod. Phys. A 27, 1250161 (2012). 